\title{
The generation of internal waves by tidal flow over continental shelf/slope topography
}

\author{
K. Lim, G.N. Ivey and R.I. Nokes ${ }^{1}$ \\ School of Environmental Systems Engineering, University of Western Australia, 35 Stirling \\ Hwy, Crawley 6009 Australia \\ ${ }^{1}$ Department of Civil and Natural Resources Engineering, University of Canterbury, \\ Christchurch, New Zealand \\ klim@sese.uwa.edu.au, ivey@sese.uwa.edu.au,roger.nokes@canterbury.ac.nz
}

\begin{abstract}
Experiments were performed to investigate the generation of internal waves induced by a barotropic tidal flow over continental shelf/slope topography in a twolayer stratified fluid. The interaction of the barotropic tide with the continental slope resulted in the formation of both linear and nonlinear waveforms, ranging from a linear wave of depression to a highly nonlinear internal bolus. The type of wave response was strongly dependent on the tidal forcing intensity and the position of the density interface relative to the shelf depth. Based on the values of the internal Froude number and the layer depth ratio, we delineate four distinct generation regimes, each with a distinct wave response.
\end{abstract}

Keywords Internal waves, internal Froude number, flow over topography, continental shelf, laboratory experiment, stratified fluid, generation, internal bolus

\section{Introduction}

Internal waves with astronomical tidal periods, or internal tides, generated by the action of the tide sweeping stratified water over non-uniform topography play an important role in deep ocean mixing and large-scale ocean circulation $[18,28]$. Over the past decades internal tides have been frequently reported near regions of non-uniform topography, for example near continental slopes [2,7], seamounts [15,24] and mid-ocean ridges [4,23]. The subsequent evolution of the internal tide into smaller scale nonlinear internal waves has been discussed in field studies [10,19], theoretical analyses [27], laboratory experiments $[1,13]$ and numerical simulations $[9,17]$.

A region of interest motivating the work in this article is the Australian North West Shelf (NWS), where observations $[6,7,10]$ show long internal tides, with wavelengths 
of approximately $20 \mathrm{~km}$, evolving into internal solitary waves and other strongly nonlinear wave forms. During the late summer months (March to April) when the vertical density gradient is the strongest, some of the largest nonlinear internal waves, with amplitudes of up to 80 meters in water of $125 \mathrm{~m}$ depth, are observed [25].

Tidal flow over a single topographic feature such as a sill has been shown to form a depression of the density interface on the lee side of the sill during the ebb phase and, as the tidal current slackens and then turns, this wave of depression advances over the topography $[13,16]$. For continental shelf/slope topography, on the ebb phase a depression is again formed near the shelf break and, as the ebb flow weakens, the depression subsequently splits into two oppositely-directed waves of depression [22]. As the wave of depression propagates, it can steepen and disperse to eventually form a train of rank ordered solitary waves. Very strong tidal flows can cause such rapid downslope flow over the topography that the depression becomes unstable and an internal hydraulic jump can form on the lee side [1] causing small-scale instabilities and nonlinear waves to develop upstream of the topographic crest [5].

While previous work has shown that generation is influenced by the form of the density stratification, the shape of the topography, and the intensity of the tidal forcing, there appears neither a general description of the generation mechanism nor a classification system to show how these independent parameters can interact and produce different types of internal wave response. We use laboratory experiments to investigate the formation of internal waves over continental slope/shelf topography and propose a set of nondimensional parameters to predict the type of wave response to be expected over the slope region. Here we focus on an idealised two-layer stratification; the generation of internal waves in a continuous stratification is discussed elsewhere [14].

\section{System Parameters}

Consider the idealised shelf/slope configuration shown in Fig.1 with an upper and lower layer, $h_{1}$ and $h_{2}$, consisting of densities $\rho_{1}$ and $\rho_{2}$, respectively, where the total depth $H=h_{1}$ $+h_{2}$. We define $\Delta \rho=\rho_{2}-\rho_{1}$ and $\rho_{0}=\left(\rho_{2}+\rho_{1}\right) / 2$ to form the reduced gravity term $g^{\prime}=g \Delta \rho / \rho_{0}$ and the barotropic flow $u=U_{0} \sin \omega t$, where $\omega$ is the tidal frequency and $U_{0}$ is the maximum barotropic velocity at the top of the slope. 


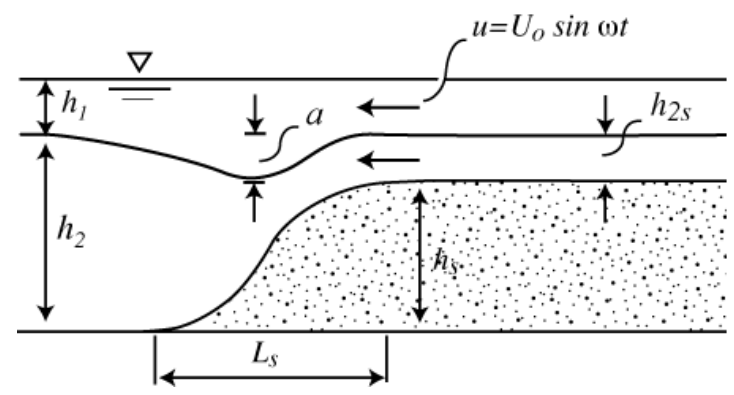

Fig. 1 Schematic diagram of the generation process

On the ebb phase, the downward displacement $a$ of the density interface can be written as

$$
a=f\left(g^{\prime}, U_{0}, \omega, h_{1}, h_{2 s}, h_{s}, L_{s}\right)
$$

where $f$ is a function to be determined and we assume negligible mixing effects and a sharp density interface. Note we confine our attention to the case where the density interface sits at or above the shelf, and thus $h_{2}$ is not a parameter in (1). Using Buckingham's PI-theorem, a suitable choice of nondimensionalised parameters is

$$
\frac{a}{h_{2 s}}=f\left(F r, \frac{U_{0} / \omega}{L_{s}}, \frac{h_{s}}{L_{s}}, \beta, \frac{h_{2 s}}{h_{s}}\right) .
$$

Here $\operatorname{Fr}=U_{0} / \sqrt{g^{\prime} h_{E}}$ is the internal Froude number, $\alpha=U_{0} / \omega L_{s}$ is the tidal excursion parameter, $S=h_{s} / L_{s}$ is the bottom slope, $h_{2 s} / h_{s}$ is the ratio of the lower shelf thickness to the shelf height, and we define $h_{E}=h_{1} h_{2 s} /\left(h_{1}+h_{2 s}\right)$ and $\beta=h_{1} /\left(h_{1}+h_{2 s}\right)$.

Motivated by the Australian NWS conditions, we are interested here in the regimes where $\mathrm{Fr} \leq 1, \alpha<<1$ and $h_{2 s} / h_{s}<<1$. If the tidal excursion is small $(\alpha<<1)$ then only the local bottom slope $S$ over which the tidal excursion occurs is the relevant slope scale, and not over the entire continental slope as given by $h_{s} / L_{s}$. Moreover it is the upper part of the continental slope that the density interface will "sense" for the cases where the density interface sits near or above the shelf and here the bottom slope $S<<1$. In these limits all three parameters $\alpha, h_{2 s} / h_{s}$ and $S$ are small and near constant, hence (2) simplifies to a twoparameter problem

$$
\frac{a}{h_{2 s}}=f(F r, \beta)
$$

which provides a framework to interpret the experimental results below. 


\section{Experimental Setup}

The experiments were performed in a sealed rectangular glass-walled tank $(5,870 \mathrm{~mm}$ long, $530 \mathrm{~mm}$ wide and $600 \mathrm{~mm}$ high) into which a shelf/slope topography, constructed out of black polyethylene sheets, was inserted across the full width of the tank (Fig. 2). The shelf section consisted of a horizontal surface 3,600 $\mathrm{mm}$ long and $300 \mathrm{~mm}$ above the tank floor. The slope section extended $800 \mathrm{~mm}$ from the edge of the shelf (the shelf break). At the end of the shelf several layers of $10 \mathrm{~mm}$ thick filter foam were used to dissipate any incoming waves and to eliminate any back reflection off the end wall. The slope section has an approximate hyperbolic tangent shape with a local bottom slope of $45^{\circ}$ at the mid point. At the other end of the tank a large isosceles-triangular plunger was connected via two guiding rods and a ball-lead screw to a precision DC servo electric motor, which oscillated the plunger vertically over a range of amplitudes and frequencies. This plunger generated a barotropic horizontal flow offshore, similar to the method used in Baines and Fang [1], and the magnitude of the induced horizontal velocity was checked with vertical profiles taken with an Acoustic Doppler Velocimeter (ADV).

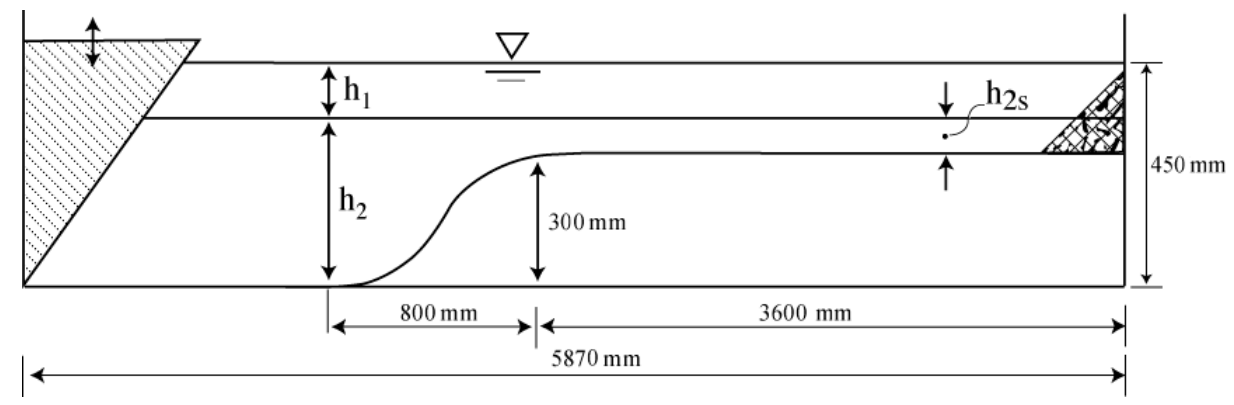

Fig. 2 Schematic diagram of experimental tank setup

The tank was then filled with a two-layer stratification to a total water depth of $450 \mathrm{~mm}$. Depending on the flow visualization method used, the tank was either filled with fresh water on top of a denser sodium chloride salt solution, or two layers of salt solutions of differing densities, giving a hyperbolic tangent density profile within a thin interfacial layer separating the upper and lower layers. For all the experiments, the density difference $\left(\Delta \rho / \rho_{0}\right)$ between the upper and lower layers was kept relatively small and constant ranging between $0.46 \%$ and $0.55 \%$.

The flow visualisation techniques, light attenuation (LA) and particle tracking velocimetry (PTV), were employed to obtain high resolution two-dimensional density and velocity fields, respectively. For the LA method, a $3 \mathrm{~mm}$ opaque Perspex diffusion sheet 
was fitted on the back face of the tank and a light bank of 26 horizontally oriented triphosphor twin $36 \mathrm{~W} 100 \mathrm{~Hz}$ fluorescent tubes with reeded diffusers was mounted $220 \mathrm{~mm}$ behind the Perspex sheet (Fig. 3). The Perspex diffusion sheet, reeded diffusers and the light bank created a uniform light sheet which completely illuminated the tank's viewing window. On the opposite side of the tank, a tripod-mounted 6.1 megapixel digital Nikon D50 SLR camera was positioned 3,500 $\mathrm{mm}$ from the front face. The field of view covered the region from the toe of the continental slope to the first $430 \mathrm{~mm}$ of the continental shelf, giving a spatial resolution of approximately $0.55 \mathrm{~mm}$ per pixel in both the horizontal and vertical directions. Digital images at 2256 x 1496 pixels were captured at 2.5 frames per second.

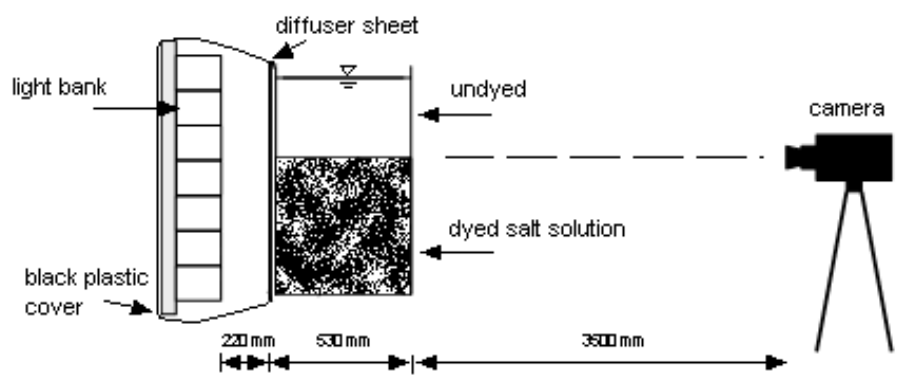

Fig. 3 Schematic diagram of Light Attenuation technique setup

The LA technique utilizes the exponential relationship between dye concentration (in this case Exacol Carmoisine red food dye) and attenuation of either the green or blue light signals. This relationship can be expressed as a simple equation for low dye concentrations [3]. With this relationship dye concentration can then act as a proxy for fluid density provided strict control of temperature in the darkened laboratory is maintained [12]. Shutter-speed, aperture, and white balance of the digital camera were preset to optimize the intensity range; errors associated with temperature and light fluctuations after calibration were less than $5 \%$. The LA results for a quiescent two-layer fluid were compared to the density profile obtained using a vertically traversed fast-response conductivity and temperature (CT) probe (precision measurement engineering, PME); the agreement between the two methods was excellent.

The two-dimensional velocity field was measured using PTV. The fluid was seeded locally with pliolite VT resin particles of diameters ranging between 180 to $250 \mu \mathrm{m}$ and illuminated with a thin light sheet projected from a $2 \mathrm{~kW}$ halogen light box, positioned at the mid-section above the surface of the tank. The emitted light is passed through two long narrow slits to form a $10 \mathrm{~mm}$ wide light sheet and the illuminated flow fields 
measuring 400 x $400 \mathrm{~mm}$ were recorded using a progressive scan CCD camera (PULNIX TM-1040, Navitar ST16160 lens) at a rate of $30 \mathrm{~Hz}$ with no shutter. The acquired digital images (resolution of 2.48 pixels per mm) were captured in a LABVIEW environment using a digital frame grabber (National Instruments PCI-1422) and written in real-time to disk.

In the PTV technique the particles are matched from frame to frame using a variety of costing strategies [21] and particle velocities are calculated based on the particle displacement and the time step between frames. To produce time series of two-dimensional velocity fields the particle velocities were interpolated onto a rectangular grid using a Thessian triangulation method. The processing and the analysis of the digital images collected from the LA and PTV experiments was performed using the software program ImageStream Version 5 [20] and Fluid Stream Version 7.01 [21], respectively.

The experimental variables for each run are detailed in Table 1 . The internal Froude number ranged from 0.11 to 0.98 . For each depth ratio, a set of experiments was run with increasing barotropic forcing frequency which resulted in a gradual thickening of the density interface from an initial thickness of approximately $20 \pm 2 \mathrm{~mm}$ to approximately $30 \pm 2 \mathrm{~mm}$. Before running each experiment the density structure was measured by vertically traversing a fast response CT probe through the fluid.

Table 1 Summary of experiments performed

\begin{tabular}{lllllllll}
\hline Run & $h_{1} / H$ & $\beta$ & $g^{\prime}$ & $\alpha$ & $F r$ & $a / \lambda$ & $a / h_{2 s}$ & Observed waveforms \\
\hline $1-4$ & 0.11 & 0.33 & 53 & $0.088-0.115$ & $0.21-0.97$ & $0.044-0.053$ & $0.14-0.20$ & Wave of depression \\
$5-8$ & 0.17 & 0.50 & 49 & $0.086-0.112$ & $0.20-0.92$ & $0.051-0.061$ & $0.19-0.37$ & Wave of depression \\
9 & 0.22 & 0.67 & 49 & 0.090 & 0.11 & - & 0.20 & Wave of depression \\
10 & 0.22 & 0.67 & 49 & 0.080 & 0.20 & 0.0357 & 0.80 & Internal surge, internal bolus \\
$11-13$ & 0.22 & 0.67 & 49 & $0.090-0.113$ & $0.33-0.98$ & $0.020-0.228$ & $0.72-0.74$ & Internal surge, solitary waves \\
14 & 0.33 & 1.00 & 44 & 0.089 & 0.07 & - & - & No waves \\
$15-17$ & 0.33 & 1.00 & 44 & $0.085-0.095$ & $0.13-0.36$ & - & - & Internal bolus \\
18 & 0.33 & 1.00 & 44 & 0.114 & 0.60 & - & - & No waves \\
$19-21$ & 0.44 & - & 60 & $0.086-0.096$ & $0.11-0.30$ & - & - & Internal bolus \\
22 & 0.44 & - & 60 & 0.112 & 0.50 & - & - & No waves \\
\hline
\end{tabular}

Note. units for $g$ ' in $\mathrm{mm} / \mathrm{s}^{2}$ 


\section{Experimental Results}

The downward displacement of the density interface $a$ was measured using Fluidstream by tracking individual particles along the density interface through the entire ebb phase and observing the particles' maximum vertical displacement. Wave characteristics, such as phase speed and wavelength, were obtained from PTV velocity field information which was processed using image pairs taken $1 / 30$ s apart. The barotropic velocity $u_{0}$ was defined as the depth-averaged horizontal velocity $u_{0}(x, t)=1 / h \int_{-h}^{0} u(x, z, t) d z$, and the baroclinic velocity $u^{\prime}(x, z, t)$ was obtained by subtracting $u_{0}$ from the total horizontal velocity $u(x, z, t)$ at each point in the domain for each time step. An example is shown in Fig. 4a,b.

We also compute the quantity, $I=\int_{-h}^{0}\left|u^{\prime}(x, z, t)\right| d z$, and from this quantity $I$ we fit a Gaussian function to the curve to obtain quantitatively the position (i.e., centroid $C_{\mathrm{i}}$ located at $I$ maximum) and width $\lambda$ of the baroclinic wave (i.e., taken as two standard deviations from either side of $C_{i}$ ) (Fig. 4c). We are thus able to measure wavelength and speed of wave propagation by tracking the position of this Gaussian distribution over the course of the experiment after removing the influence of the background flow. With estimates of $a$ and $\lambda$, we can thus compute wave steepness $a / \lambda$. Note in the experimental runs which have either very weak forcing or very strong forcing it was difficult to estimate $\lambda$ reliably due to the weak baroclinic signature. 
a)

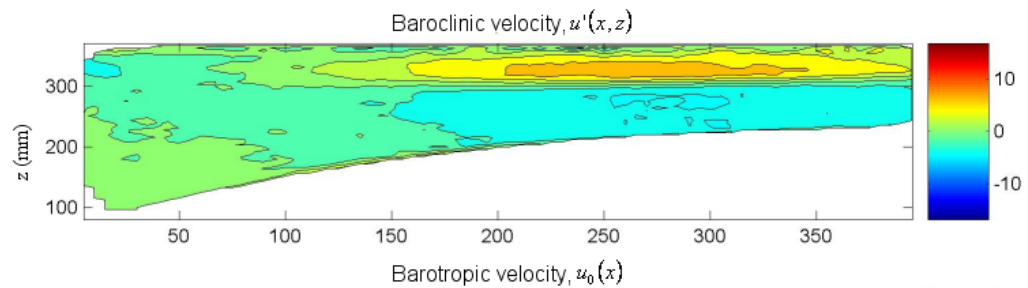

b)

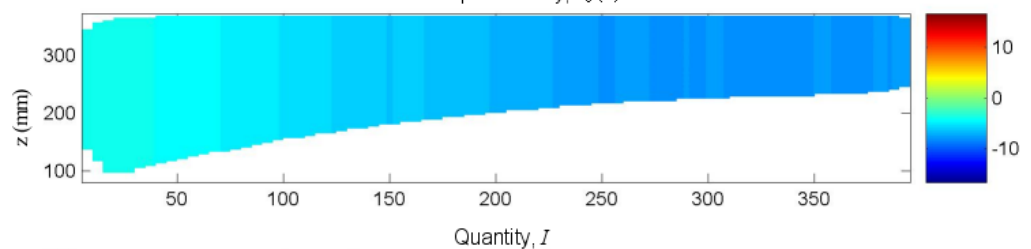

c)

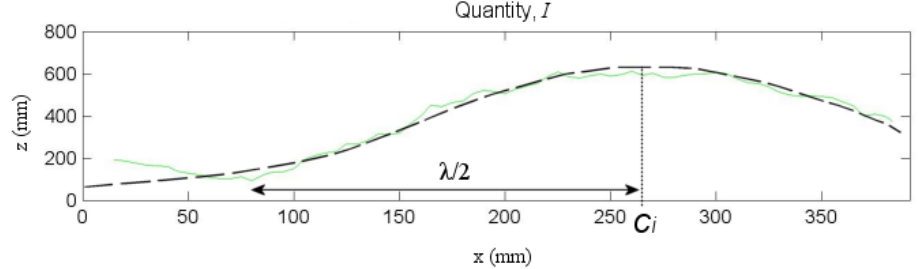

Fig. 4 Baroclinic velocity $u$ '(mm/s), barotropic velocity $u_{0}(\mathrm{~mm} / \mathrm{s})$ and quantity $I$ computed over a section of the continental slope at a particular time

It is convenient to present the experimental results in three distinct categories based on the magnitude of the layer depth ratio: (i) $\beta<0.5$ (ii) $0.5<\beta<1$ (iii) $\beta=1$.

\section{$4.1 \beta<0.5$}

A typical run is shown in Fig. 5. On the ebb phase a simple depression of the interface forms over the upper section of the slope. As the ebb current increases to a maximum value, the amplitude and width of this depression increases (Fig. 5a). As the ebb flow weakens and turns, there was little evidence of offshore wave propagation but a single wave of depression, of unchanging form, was clearly propagating onshore (Fig. 5b). 
a)

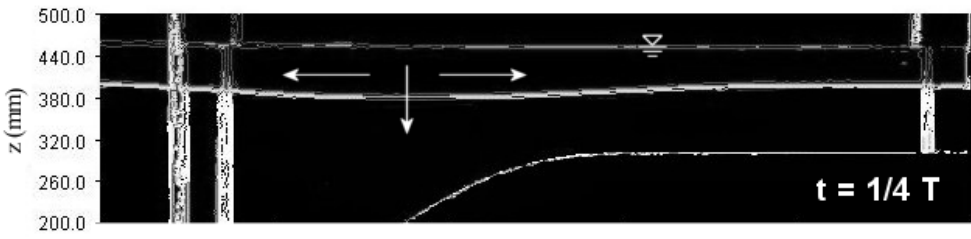

b)

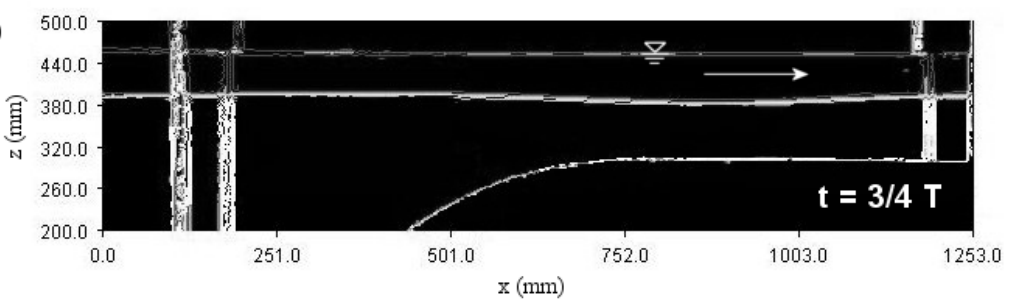

Fig. 5 Sequence of LA images (a,b) showing generation and propagation of a small amplitude wave of depression during half a tidal cycle T, for Run $2-\beta=0.33$ and $F r=0.32$

When the background flow was weak $(F r<0.5)$, the depression was able to propagate onto the shelf. However, as the intensity of the barotropic forcing increased to $F r>0.5$, the depression was arrested during part of the ebb phase and its shape was slightly distorted by the background flow (Runs 3 and 4). At $F r=0.98$, mixing was observed at the trough of the depression as the barotropic flow reversed from ebb to flood phase causing strong shear across the density interface.

For all runs the amplitude $a$ of the wave was small compared to the depth of the lower shelf layer $\left(a / h_{2 s}<0.4\right)$ with little apparent dependence on $F r$. Estimates of wave steepness were less than 0.1 which suggests the wave has linear characteristics. However, the onshore phase speed of the depression was measured to be approximately half the magnitude expected from linear long-wave theory for free waves. Finally, the observed wavelength of the baroclinic wave, to an accuracy of $\pm 10 \%$, was given by $4 U_{0} / \omega$, that is twice the barotropic tidal horizontal excursion length.

\section{$4.20 .5<\beta<1$}

A typical run is shown in Fig. 6. Like the previous case, a depression formed over the upper part of the slope during the ebb phase but it is asymmetrical due to the acceleration of flow in the bottom layer resulting in a slight steepening of the trailing face of the depression (Fig. 6a). As the ebb flow turns to flood, the trailing face steepened further forming an internal surge (as termed by Horn et al. [11] to describe the formation of a steep fronted wave due to nonlinear steepening) (Fig. 6b) before transforming into a packet of solitary waves of elevation, arranged by amplitude, as can be seen in Fig. 6c,d. 


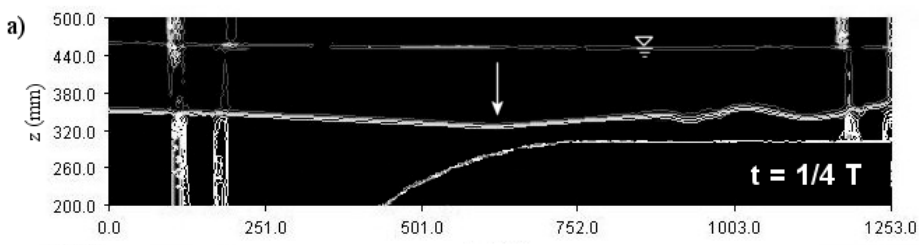

b)
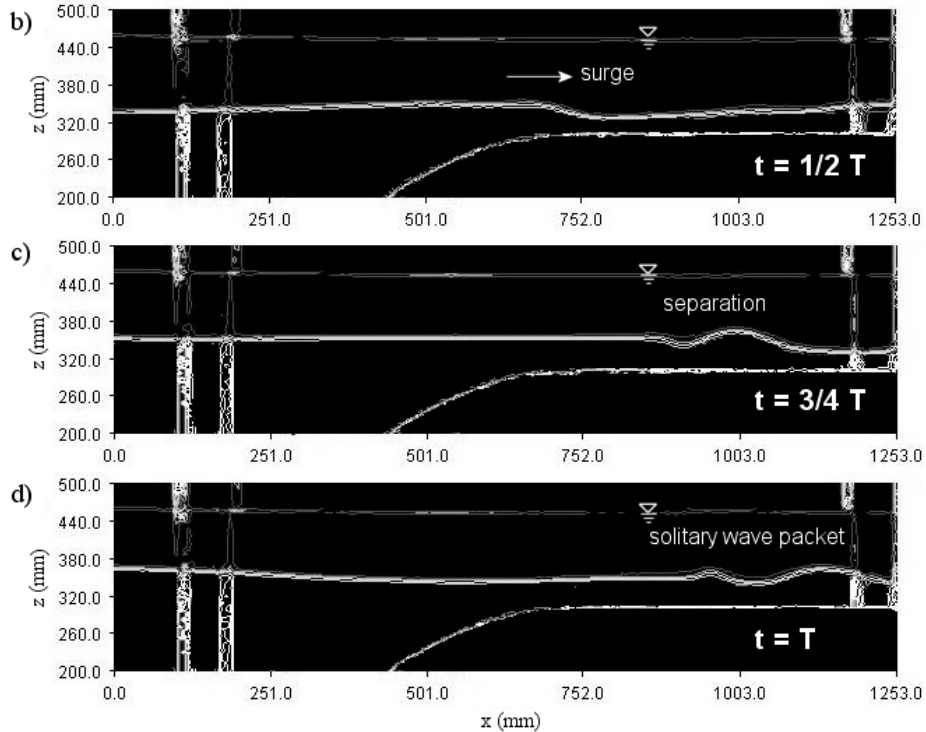

Fig. 6 Sequence of LA images (a-d) showing steepening of wave front and subsequent evolution into packet of solitary waves during one tidal cycle $\mathrm{T}$, for Run $11-\beta=0.67$ and $\mathrm{Fr}=0.33$

For very weak barotropic forcing $(F r=0.11)$ the depression was small $\left(a / h_{2 s}=0.2\right)$ and remained as a depression waveform as it propagated onshelf. For stronger flows $(F r=0.20$ to 0.98$)$ the trough of the depression reached close to the slope $\left(a / h_{2 s}>0.7\right)$, resulting in the steepening and subsequent formation of a nonlinear surge. A second example is shown in Fig. 7. In this example, the trough of the depression reached almost to the bottom $\left(a / h_{2 s}=\right.$ 0.8) causing strong acceleration of flow in the lower layer, resulting in an internal hydraulic jump and the formation of a vortex instability (referred to as an internal bolus by Venayagamoorthy and Fringer [26]) behind the wave front. 
a)

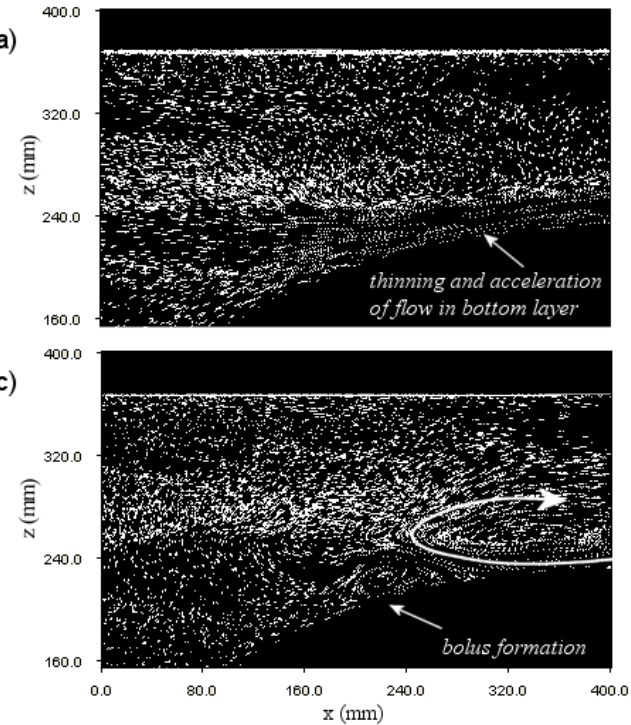

b)

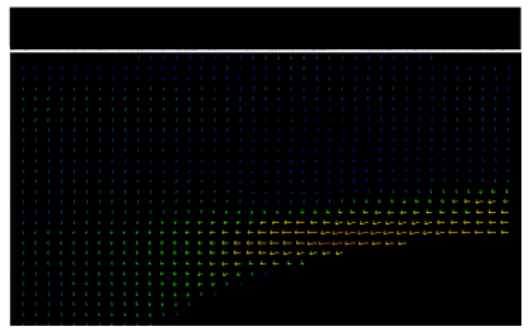

d)

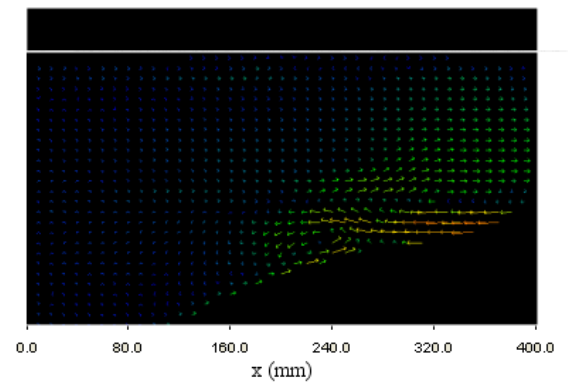

Fig. 7 Sequence of particle tracking images (a,c) and their corresponding velocity vector field (b,d), showing the formation of an internal bolus, for Run $10-\beta=0.67$ and $F r=0.20$. Each particle image displays 5 consecutive particle frames.

In Fig. 7a,b the thinning and acceleration of the bottom layer is clearly observed during the ebb phase. Soon afterwards, the flow in the bottom layer becomes supercritical, leading to an internal hydraulic jump and resulting in active overturning of the isopycnals near the bottom slope. As the barotropic flow reverses, flow of lighter fluid continues to descend from upslope and is entrained under the denser fluid. The interaction between the strong downslope flow with the locally gravitationally unstable fluid generates a vortex instability (rotating anticlockwise) or an internal bolus (Fig. 7c,d). The greater the entrainment of lighter fluid under the denser fluid, the more energetic, faster rotating and the larger the internal bolus becomes. The internal bolus continues to propagate upslope behind the internal surge on the flood phase onto the shelf.

\section{$4.3 \beta=1$}

A representative run is shown in Fig. 8. During the ebb phase the density interface is drawn down by the strong downslope flow (Fig. 8a). The downslope flow continues to accelerate on the ebb causing the interface to steepen further until it becomes statically unstable, it breaks, and lighter upper layer fluid is entrained into the denser fluid, (Fig. 8b). The process of breaking and entrainment of lighter fluid forms an internal bolus of mixed fluid which, as the ebb flow weakens, surges onto the shelf (Fig. 8c). The bolus continues to propagate 
forward onto the shelf where it slows and eventually dissipates (Fig. 8d). The process repeats itself each tidal cycle, although as the region of generation becomes more mixed, the resulting bolus continues to force its way onto the shelf but is less dense than the previous bolus. This scenario is consistent with the numerical observations made by Venayagamoorthy and Fringer [26].

a)

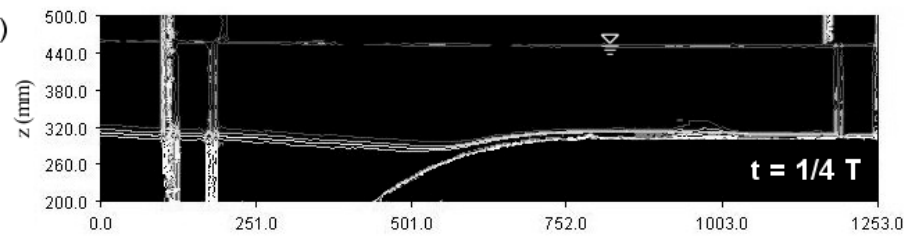

b)

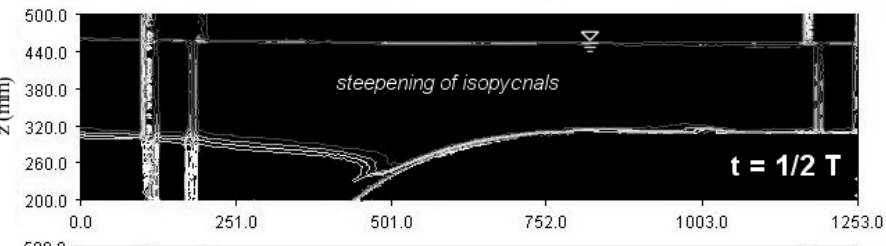

c)

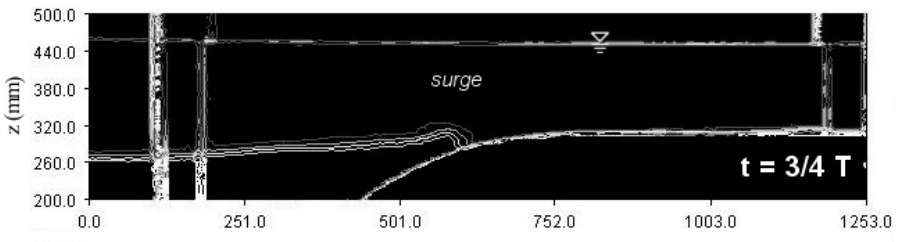

d)

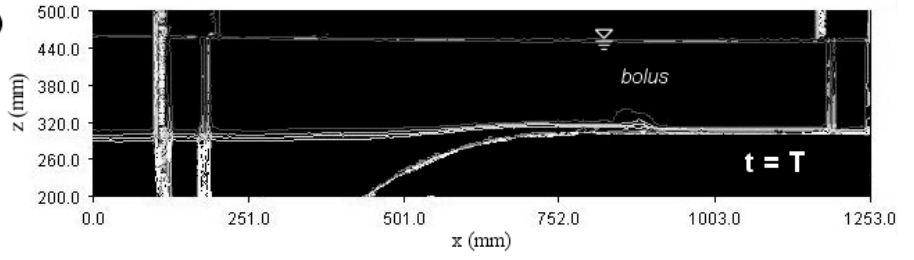

Fig. 8 Sequence of LA images (a-d) showing formation of an internal bolus during one tidal cycle $\mathrm{T}$, for Run $16-\beta=1, F r=0.20$

For weak forcing $(F r<0.1)$ there was little vertical displacement of the isopycnals and no waves were observed. For $\mathrm{Fr}>0.3$ the bolus was arrested by the ebb flow and was not able to propagate past the slope region. For $F r>0.5$, strong mixing is observed on the slope region due to shearing across the density interface. Thus, in order to generate internal boluses the system needs to be forced at an optimal forcing intensity $(0.1<F r<0.3)$, where beyond this range wave motions are either damped or rapidly dissipated through mixing and accelerations or decelerations associated with the background barotropic flow. 


\section{Discussion}

The generation process occurs over a tidal period, $\mathrm{T}=2 \pi / \omega$, hence the generation timescale $T_{g}$ is defined as,

$$
T_{g} \sim 1 / \omega
$$

The generation region may be considered as extending from the slope to the point on the shelf to which the wave has propagated at $\mathrm{t}=\mathrm{T}$ (see Fig. 5, 6, and 8). One can also define a propagation timescale $T_{p}$ for a free wave propagating onto the shelf as

$$
T_{p} \sim \frac{\lambda}{c} \sim \frac{F r_{0}}{\omega}
$$

where we have used the experimental observation that the wavelength is of the order of the tidal excursion length. Thus the ratio of these two important timescales is

$$
\frac{T_{g}}{T_{p}} \sim \frac{1}{F r_{0}}
$$

The flows in the experiments range between $F r \sim 0.1$ and $F r \sim 1$. The implication is that any waves seen are likely to be strongly affected by the generation process and are not really freely propagating waves, which explains the slower propagation speed that was observed for the linear depression wave (Sect. 4.1).

From the experimental observations the initial displacement $a$ of the wave is generated during the ebb phase. An upper bound on the displacement $a$ is found by taking the product of the induced vertical velocity $w \sim S U_{o}$ with the generation timescale $T_{g}$ which yields

$$
a_{i} \sim S \frac{U_{0}}{\omega}
$$

A lower bound may be obtained by assuming buoyancy effects control the extent of the interface displacement. From the vertical momentum equation we assume a balance between vertical advection and buoyancy to give

$$
\frac{w^{2}}{a_{b}} \sim g^{\prime}
$$

Taking $w \sim S U_{0}$, a lower bound estimate of the interface displacement $a_{b}$ is

$$
a_{b} \sim S^{2} \frac{U_{0}^{2}}{g^{\prime}}
$$


The observed values of $a$ are compared to these scale estimates in Table 2. Note the bottom slope $S$ was measured over the tidal excursion length at the top of the slope. For all the experiments the tidal excursion was small $(\alpha<0.12)$ and varied little between each run (see Table 1), and hence the bottom slope variation was small and assumed to be constant. It is clear that $a_{b}$ significantly underpredicts the observed amplitude $a$, while $a_{i}$ is a good approximation for $\beta<0.5$ and in some other cases (Run 6 and 9) where $F r$ is small (i.e., when the response is linear). The observations are plotted in Fig. 9 and as predicted by (3) the character of the waves that are generated is clearly dependent upon both $\beta$ and $F r$, with the former being the dominant factor.

Table 2 Estimates of displacement $a$ (observed), $a_{i}$ and $a_{b}$ for experimental runs 1 to 13 (Note: units for $a, a_{i}, a_{b}$ are in $\mathrm{mm}$ )

\begin{tabular}{llllll}
\hline Run & $\beta$ & $F r$ & $a$ & $a_{i}$ & $a_{b}$ \\
\hline 1 & 0.33 & 0.21 & 15 & 13 & 0.05 \\
2 & 0.33 & 0.32 & 17 & 14 & 0.12 \\
3 & 0.33 & 0.58 & 14 & 15 & 1.12 \\
4 & 0.97 & 20 & 17 & 0.05 \\
5 & 0.33 & 0.20 & 20 & 13 & 0.13 \\
6 & 0.31 & 14 & 14 & 0.43 \\
7 & 0.50 & 0.56 & 23 & 15 & 1.14 \\
8 & 0.50 & 28 & 17 & 0.01 \\
9 & 0.50 & 0.92 & 15 & 14 & 0.05 \\
10 & 0.67 & 0.11 & 40 & 12 & 0.13 \\
11 & 0.67 & 0.20 & 36 & 14 & 0.41 \\
12 & 0.67 & 0.33 & 37 & 14 & 1.16 \\
\hline 13 & 0.67 & 0.59 & 37 & 14 & \\
\hline
\end{tabular}

In terms of the overall behaviour of the response, the observations suggest we can define four regimes of wave response depending on $F r$ and $\beta$ conditions, as shown schematically in Fig. 10. Note the position of regime boundaries are best estimates from the present experimental runs (see Table 1), but also includes experimental results from Baines and Fang [1], Ramirez and Renouard [22], and field measurements taken from the North Rankin region on the Australian NWS (discussed later) [25]. The regions where the regimes overlap suggest a mixture of wave responses. 


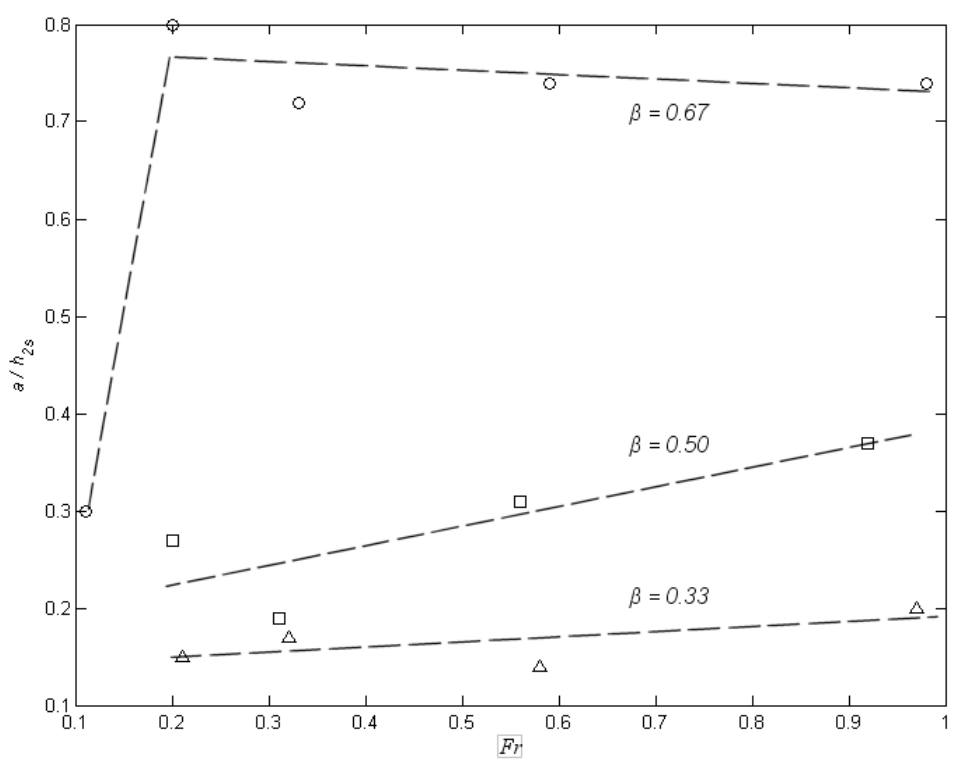

Fig. $9 \quad$ Plot of $a / h_{2 s}$ vs $F r$ for fixed depth ratio $\beta(\Delta, \beta=0.33$; $\square, \beta=0.50 ; 0, \beta=0.67)$

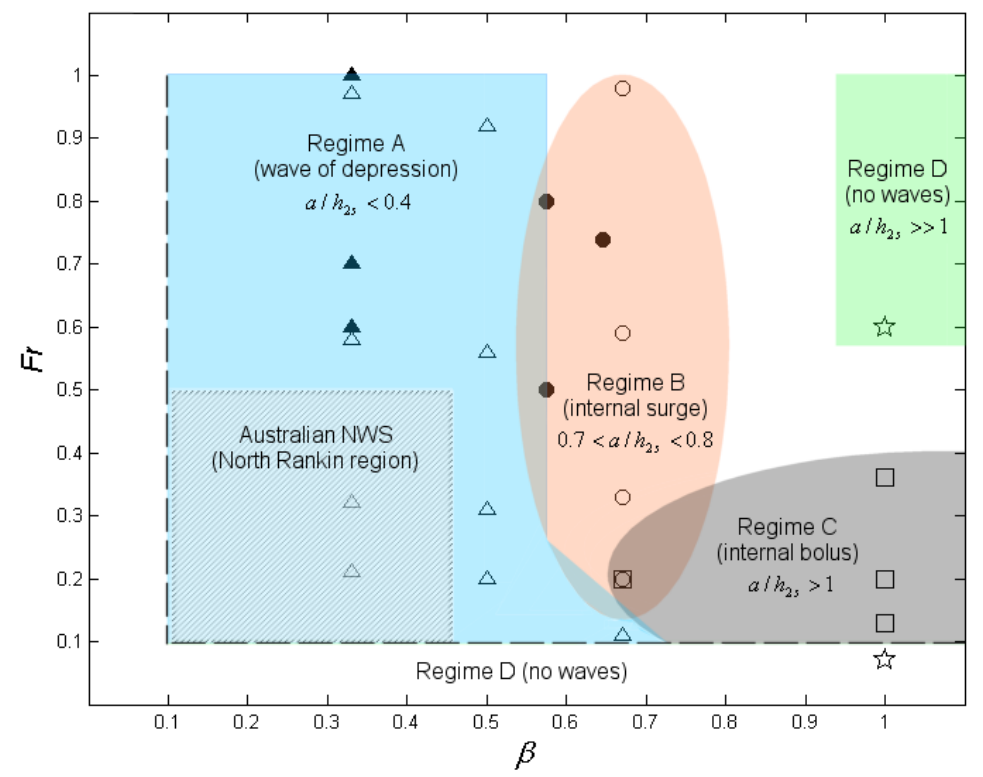

Fig. 10 The regimes of wave response over the slope for the laboratory experiments described in Sect. 4 are defined in terms of the internal Froude number $F r$ and depth ratio $\beta$ ( $\Delta$ or $\boldsymbol{\Lambda}$, wave of depression; o or $\bullet$, internal surge; $\square$, internal bolus; $\boldsymbol{\sim}$, no waves). The experimental observations from Ramirez and Renouard [22] ( $\mathbf{\Lambda}$ ) and Baines and Fang [1] $(\bullet)$ and field measurements taken from the North Rankin region on the Australian NWS [25] are also plotted. 
The parameter $a / h_{2 s}$ can be considered as a measure of nonlinearity of the wave generation process. For $a / h_{2 s}<0.4$ the wave response is linear and we only observed linear waves of depression (Regime A). For $0.4<a / h_{2 \mathrm{~s}}<0.7$ the wave response is weakly nonlinear and the wave of depression begins to exhibit characteristics of an internal surge (overlap region between Regime A and B). In the range $0.7<a / h_{2 \mathrm{~s}}<0.8$, nonlinear effects increase and only internal surges formed (Regime B). For $a / h_{2 s} \geq 0.8$, the amplitude becomes comparable to the lower layer depth on the shelf; the flow in the lower layer becomes unstable and results in the formation of an internal bolus (Regime C). Finally, when the amplitude of the displacement exceeds the lower layer depth $\left(a / h_{2 s}>>1\right)$ the density interface breaks and no waves are formed (Regime D). If the forcing is large $(F r>0.5)$ dissipation and mixing will prevent internal waves from forming. Conversely, if the tidal forcing is very weak $(F r<0.1)$, or when the surface layer is very thin, we expect friction to be important and any wave motion to be quickly damped.

\subsection{Field Observations}

To determine the applicability of the analysis to real oceanic flows over continental shelf/slope topography we examined observations from the North Rankin region, located on the Australian NWS (Fig. 11). Here, the tidal excursion is small relative to the scale of the continental slope ( $\alpha \sim 0.02$ ), the continental slope is gently sloping ( $S \sim 0.02$ ), and the earth's rotation is ignored since it does not affect the wave generation mechanism [22], a

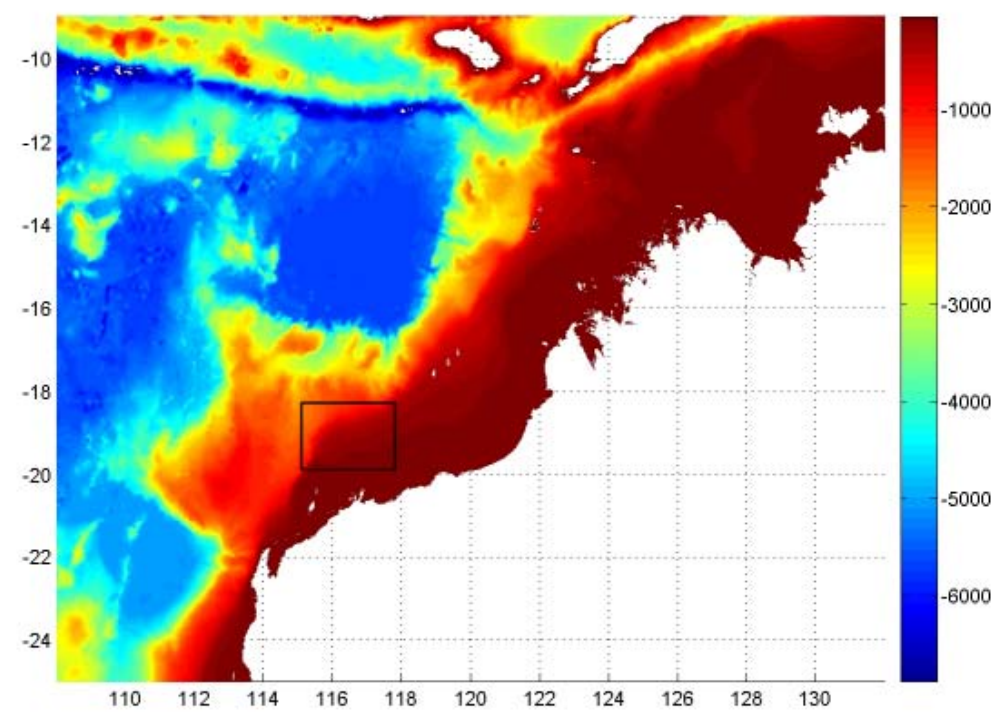

Fig. 11 Location of North Rankin region on the Australian North West Shelf 
necessary condition for (3) to be valid.

Van Gastel et. al [25] described results from a field experiment in the North Rankin region which measured current velocity and temperature data from a stationary mooring and a benthic L-shaped array in $124 \mathrm{~m}$ of water. Furthermore they applied the Regional Ocean Modelling System (ROMS) model over an $800 \mathrm{~km}$ by $500 \mathrm{~km}$ domain to characterize the physical oceanography in the region. Their temperature records show that the ocean is density stratified with a distinct thermocline throughout the months of March to May 2004. The position of the thermocline changes from $20 \mathrm{~m}$ depth in March to $90 \mathrm{~m}$ depth in May due to the combination of seasonal heating/cooling and wind-driven mixing processes. During this period, highly nonlinear waves of depression were only observed when the thermocline was positioned above mid-depth, and only waves of elevation were observed when the thermocline was below mid depth [25], which agrees with the experimental observations in this paper (see Fig. 5 and 6). The authors suggest the waves were generated over the continental slope in water depths of 400 to $500 \mathrm{~m}$, approximately $70 \mathrm{~km}$ from the measurement location [25]. We consider the continental slope to begin at a water depth of $200 \mathrm{~m}$, approximately $20 \mathrm{~km}$ from the mooring location, and extending to more than $2000 \mathrm{~m}$ deep. Numerical simulations were run for 60 days from $13^{\text {th }}$ March 2004 and predicted peak barotropic tidal currents ranging from $0.1 \mathrm{~m} / \mathrm{s}$ (neap tide) to $0.25 \mathrm{~m} / \mathrm{s}$ (spring tide) at the $200 \mathrm{~m}$ water depth, which are similar to the observed and modelled tidal ellipse magnitudes presented in [8]. We estimate the internal Froude number $\mathrm{Fr}$ to range from 0.1 to 0.5 and the layer depth ratio $\beta$ to range from 0.1 to 0.45 . As shown in Fig. 10 the regime diagram predicts the generation of waves of depression only in the North Rankin region. The observations by Van Gastel et al. [25] generally agree except that it was shown in May that waves of elevation were also formed. The reason is simple. In reality the continental shelf is gradually sloping, unlike the configuration in our experiments where the shelf was flat. It can be imagined that a linear depression wave was first formed over the continental slope in $\sim 450 \mathrm{~m}$ of water with the thermocline positioned at $90 \mathrm{~m}$ depth (i.e. $\beta$ $\sim 0.2$ ) and as it propagates into shallower shelf waters the wave will steepen due to nonlinear effects. By the time it reaches the measurement location in $124 \mathrm{~m}$ water depth the thermocline is now positioned near the bottom (i.e. $\beta \sim 0.8$ ) and only waves of elevation are supported. An example of this evolution from a depression wave on the slope to waves of elevation on the shelf is shown in Fig. 6.

Differences between observations in the real ocean and the two-layer experiments are not unexpected. Other external parameters such as the exact form of the density stratification, variable forcing and three-dimensional topographic effects may influence the generation process, unlike the configuration in our idealised two-layer experiments. 


\section{Conclusions}

Laboratory experiments were conducted to investigate the formation of baroclinic waves by tidal flow over a continental slope in a two-layer stratified fluid. The experiments covered a range of tidal forcing intensities from subcritical to critical flow while the depth ratio was varied from a thin upper layer $(\beta=0.33)$ to one in which the density interface was positioned below the shelf height $(\beta=1)$. No prior laboratory experiments have examined cases where $\beta>0.65$.

Using density and velocity measurement techniques we were able to provide a detailed description of the complex flow fields during the generation process. We identified four distinct regimes in which a particular wave response is expected to dominate depending on both the tidal forcing intensity and the position of the density interface: (a) formation of linear depression waves; (b) formation of an internal surge; (c) formation of an internal bolus; and (d) no waves. Further work should be undertaken to determine the boundary of each of the observed wave responses in the regime diagram as a function of $\mathrm{Fr}$ and $\beta$.

Observations of internal waves on the Australian NWS were shown to compare well with the experimental results which indicate that the analysis is a useful tool for characterising real oceanic flows over continental shelf/slope topography. Observations in other continental shelf regions should be examined to strengthen the applicability of the analysis.

Acknowledgements This research work was supported by the Australian Research Council, Woodside Energy Ltd and the West Australian Marine Science Institute (WAMSI). K. Lim acknowledges the support of the Australian Postgraduate Award and R.I. Nokes, a Gledden Senior Visiting Fellowship at University of Western Australia. The authors would like to thank Stan Stroud from Woodside for his support of the project.

\section{References}

1. Baines PG, Fang XH (1985) Internal tide generation at a continental shelf/slope junction: A comparison between theory and a laboratory experiment. Dynam. Atmos. Oceans 9:297-314

2. Carter GS, Gregg MC, Lien RC (2005) Internal waves, solitary-like waves, and mixing on the Monterey Bay shelf. Cont. Shelf Res. 25:1499-1520

3. Cenedese C, Dalziel S (1998) Concentration and depth fields determined by the light transmitted through a dyed solution. paper presented at Proceedings 8th International Symposium on Flow Visualisation (ISFV '98), Sorento, Italy, June.

4. Cummins PF, Cherniawski JY, Foreman MGG (2001) North Pacific internal tides from the Aleutian Ridge: Altimeter observations and modelling. J. Mar. Res. 59:167-191 
5. Farmer DM, Armi L (1999) The generation and trapping of solitary waves over topography. Science 283:188-190

6. Holloway PE (1987) Internal hydraulic jumps and solitons at a shelf break region on the Australian North West Shelf. J. Geophys. Res. 92:5405-5416

7. Holloway PE (1994) Observations of internal tide propagation on the Australian North West Shelf. J. Phys. Oceanogr. 24:1706-1716

8. Holloway PE (2001) A regional model of the semidiurnal internal tide on the Australian North West Shelf. J. Geophys. Res. 106:19625-19638

9. Holloway PE, Merrifield MA (1999) Internal tide generation by seamounts, ridges and islands. J. Geophys. Res. 104:25937-25951

10. Holloway PE, Pelinovsky E, Talipova T, Barnes B (1997) A nonlinear model of internal tide transformation on the Australian North West Shelf. J. Phys. Oceanogr. 27:871-896

11. Horn DA, Imberger J, Ivey GN (2001) The degeneration of large-scale interfacial gravity waves in lakes. J. Fluid Mech. 434:181-207

12. Kikkert GA (2006) Buoyant jets with two and three-dimensional trajectories. PhD thesis, University of Canterbury, Christchurch, New Zealand.

13. Lansing FS, Maxworthy T (1984) On the generation and evolution of internal gravity waves. J. Fluid Mech. 145:127-149

14. Lim K, Ivey GN, Jones NL (2008) An experimental study on the tidal generation of internal waves over continental slope. J. Fluid Mech.:In prep.

15. Lueck RG, Mudge TD (1997) Topographically induced mixing around a shallow seamount. Science 276:1831-1833

16. Maxworthy T (1979) A note on the internal solitary waves produced by tidal flow over a three-dimensional ridge. J. Geophys. Res. 84:338-346

17. Merrifield MA, Holloway G, Johnston TMS (2001) The generation of internal tides at the Hawaiian Ridge. Geophys. Res. Lett. 28:559-562

18. Munk W, Wunsch C (1998) Abyssal recipes II: energetics of tidal and wind mixing. Deep-Sea Res. I 45:1977-2010

19. New AL, Pingree RD (1992) Local generation of internal soliton packets in the central Bay of Biscay. Deep-Sea Res. 39:1521-1534

20. Nokes RI (2005) Imagestream Version 5.01: System Theory and Design, Department of Civil Engineering, University of Canterbury, Christchurch

21. Nokes RI (2007) Fluidstream Version 7.01: System Theory and Design, Department of Civil Engineering, University of Canterbury, Christchurch

22. Ramirez C, Renouard D (1998) Generation of internal waves over a shelf. Dynam. Atmos. Oceans 28:107-125

23. Ray RD, Mitchum GT (1997) Surface manifestation of internal tides in the deep ocean: Observations from altimetry and island gauges. Prog. Oceanogr. 40:135162

24. Toole JM, Schmitt RW, Polzin KL (1997) Near-boundary mixing above the flanks of a midlatitude seamount. J. Geophys. Res. 102:947-959

25. Van Gastel P, Ivey GN, Meuleners M, Antenucci JP, Fringer OB (2008) Seasonal variability of the nonlinear internal wave climatology on the Australian North West Shelf. Cont. Shelf Res.:submitted

26. Venayagamoorthy SK, Fringer OB (2007) On the formation and propagation of nonlinear internal boluses across a shelf break. J. Fluid Mech. 577:137-159

27. Vlasenko V, Staschuk N, Hutter K (2005) Baroclinic tides: Theoretical modeling and observational evidence, Cambridge University Press, New York

28. Wunsch C, Ferrari R (2004) Vertical mixing, energy, and the general circulation of the oceans. Annu. Rev. Fluid Mech. 36:281-314 